\title{
SYMMETRY IN THE SEQUENCE OF APPROXIMATION COEFFICIENTS
}

\author{
AVRAHAM BOURLA
}

(Communicated by Matthew A. Papanikolas)

\begin{abstract}
Let $\left\{a_{n}\right\}_{1}^{\infty}$ and $\left\{\theta_{n}\right\}_{0}^{\infty}$ be the sequences of partial quotients and approximation coefficients for the continued fraction expansion of an irrational number. We will provide a function $f$ such that $a_{n+1}=f\left(\theta_{n \pm 1}, \theta_{n}\right)$. In tandem with a formula due to Dajani and Kraaikamp, we will write $\theta_{n \pm 1}$ as a function of $\left(\theta_{n \mp 1}, \theta_{n}\right)$, revealing an elegant symmetry in this classical sequence and allowing for its recovery from a pair of consecutive terms.
\end{abstract}

\section{INTRODUCTION}

Given an irrational number $r$ and a rational number written as the unique quotient $\frac{p}{q}$ of the two integers $p$ and $q$ with $\operatorname{gcd}(p, q)=1$ and $q>0$, our fundamental object of interest from diophantine approximation is the approximation coefficient $\theta\left(r, \frac{p}{q}\right):=q^{2}\left|r-\frac{p}{q}\right|$. Small approximation coefficients suggest high quality approximations, combining accuracy with simplicity. For instance, the error in approximating $\pi$ using the fraction $\frac{355}{113}=3.14159203539823008849557522124$ is smaller than the error of its decimal expansion to the fifth digit, $3.14159=\frac{314159}{100000}$. Since the former rational also has a much smaller denominator, it is of far greater quality than the latter. Indeed $\theta\left(\pi, \frac{355}{113}\right)<0.0341$, whereas $\theta\left(\pi, \frac{314159}{100000}\right)>26535$.

We obtain the high quality approximations for $r$ by using the euclidean algorithm to write $r$ as an infinite continued fraction:

$$
r=a_{0}+\left[a_{1}, a_{2}, \ldots\right]:=a_{0}+\frac{1}{a_{1}+\frac{1}{a_{2}+\ldots,}}
$$

where the partial quotients $a_{0}=a_{0}(r) \in \mathbb{Z}$ and $a_{n}=a_{n}(r) \in \mathbb{N}:=\mathbb{Z} \cap[1, \infty)$ for all $n \geq 1$ are uniquely determined by $r$. This expansion also provides us with the infinite sequence of rational numbers

$$
\frac{p_{0}}{q_{0}}:=\frac{a_{0}}{1}, \quad \frac{p_{n}}{q_{n}}:=a_{0}+\left[a_{1}, \ldots, a_{n}\right], \quad n \geq 1,
$$

tending to $r$ known as the convergents of $r$. Define the approximation coefficient of the $\mathrm{n}^{\text {th }}$ convergent of $r$ by

$$
\theta_{n}:=\theta\left(r, \frac{p_{n}}{q_{n}}\right)=q_{n}^{2}\left|r-\frac{p_{n}}{q_{n}}\right|
$$

Received by the editors October 16, 2011 and, in revised form, December 1, 2011 and December 8, 2011.

2010 Mathematics Subject Classification. Primary 11A55, 11J70, 11J71, 11J81; Secondary 01-02. 
and refer to the sequence $\left\{\theta_{n}\right\}_{0}^{\infty}$ as the sequence of approximation coefficients. Since adding an integer to a fraction does not change its denominator, the number $x_{0}:=r-a_{0}$ shares the same sequences $\left\{a_{n}\right\}_{1}^{\infty}$ and $\left\{\theta_{n}\right\}_{0}^{\infty}$ as $r$, allowing us to restrict our attention solely to the unit interval. Throughout this paper, we fix an initial seed $x_{0} \in(0,1)-\mathbb{Q}$ and let $\left\{a_{n}\right\}_{1}^{\infty}$ and $\left\{\theta_{n}\right\}_{0}^{\infty}$ be its sequences of partial quotients and approximation coefficients. While the rest of this section is not a prerequisite, the following results illustrate some of the key properties for this classical sequence and are given for motivation as well as for sake of completeness.

For all $n \geq 0$, it is well known [2, Theorem 4.6] that

$$
\left|x_{0}-\frac{p_{n}}{q_{n}}\right|<\frac{1}{q_{n} q_{n+1}}<\frac{1}{q_{n}^{2}} .
$$

We conclude that $\theta_{n}<1$ for all $n \geq 0$. Conversely, Legendre 2, Theorem $5.12]$ proved that if $\theta\left(x_{0}, \frac{p}{q}\right)<\frac{1}{2}$, then $\frac{p}{q}$ is a convergent of $x_{0}$. In 1891, Hurwitz proved that there exist infinitely many pairs of integers $p$ and $q$, such that $\theta\left(x_{0}, \frac{p}{q}\right)<\frac{1}{\sqrt{5}} \approx 0.4472$ and that this constant, known as the Hurwitz Constant, is sharp. Therefore, all irrational numbers possess infinitely many high quality approximations using rational numbers whose associated approximation coefficients are less than $\frac{1}{\sqrt{5}}$. Using Legendre's result, we see that all these high quality approximations must belong to the sequence of continued fraction convergents for $x_{0}$.

We may restate Hurwitz's theorem as the sharp inequality $\liminf _{n \rightarrow \infty}\left\{\theta_{n}\right\} \leq \frac{1}{\sqrt{5}}$. In general, we use the value of $\liminf _{n \rightarrow \infty}\left\{\theta_{n}\right\}$ to measure how well $x_{0}$ can be approximated by rational numbers. The set of values taken by $\liminf _{n \rightarrow \infty}\left\{\theta_{n}\left(x_{0}\right)\right\}$, as $x_{0}$ varies in the set of all irrational numbers in the interval, is called the Lagrange Spectrum, and those irrational numbers $x_{0}$ which construe the spectrum, that is, for which $\liminf _{n \rightarrow \infty}\left\{\theta_{n}\left(x_{0}\right)\right\}>0$, are called badly approximable numbers. It is known 2 , Theorem 7.3] that $x_{0}$ is badly approximable if and only if its sequence of partial quotients $\left\{a_{n}\right\}_{1}^{\infty}$ is bounded. For more details about the Lagrange Spectrum, refer to 3 .

In 1895, Vahlen [4, Corollary 5.1.13] proved that for all $n \geq 1$ we have the sharp inequality

$$
\min \left\{\theta_{n-1}, \theta_{n}\right\}<\frac{1}{2},
$$

and in 1903, Borel [4, Theorem 5.1.5] proved the sharp inequality

$$
\min \left\{\theta_{n-1}, \theta_{n}, \theta_{n+1}\right\}<5^{-.5} .
$$

More recent improvements include the sharp inequalities

$$
\min \left\{\theta_{n-1}, \theta_{n}, \theta_{n+1}\right\}<\left(a_{n+1}^{2}+4\right)^{-.5}
$$

and

$$
\max \left\{\theta_{n-1}, \theta_{n}, \theta_{n+1}\right\}>\left(a_{n+1}^{2}+4\right)^{-.5},
$$

due to Bagemihl and McLaughlin [1] and Tong [7]. Therefore, this sequence exhibits a bounding symmetry on a triple of consecutive terms, which stems from its internal connection with the sequence of partial quotient. 
For instance, we write

$$
\pi-3=\frac{1}{7+\frac{1}{15+\frac{1}{1+\frac{1}{292+\ldots}}}}=[7,15,1,292,1,1,1,2,1,3,1,14,2, \ldots]
$$

The first ten convergents $\left\{\frac{p_{n}}{q_{n}}\right\}_{0}^{9}$ are

$$
\left\{\frac{0}{1}, \frac{1}{7}, \frac{15}{106}, \frac{16}{113}, \frac{4687}{33102}, \frac{4703}{33215}, \frac{9390}{66317}, \frac{14093}{99532}, \frac{37576}{265381}, \frac{51669}{364913}\right\},
$$

and the best upper bounds for $\left\{\theta_{n}\right\}_{0}^{9}$ using a four digit decimal expansion are

$$
\{0.1416,0.0612,0.9351,0.0034,0.6237,0.3641,0.5363,0.2885,0.6045,0.2134\} \text {. }
$$

In particular, the small approximation coefficient $\theta_{3}=0.0034$ helps explain why the rational number $\frac{355}{113}=3+\frac{16}{113}$, first discovered by Archimedes (c. 287-212 BC), was a popular approximation for $\pi$ throughout antiquity.

\section{Preliminary Results}

In 1921, Perron [6] proved that

$$
\frac{1}{\theta_{n-1}}=\left[a_{n+1}, a_{n+2}, \ldots\right]+a_{n}+\left[a_{n-1}, a_{n-2}, \ldots, a_{1}\right], \quad n \geq 1,
$$

where we take $[\emptyset]:=0$ when $n=1$. Thus, as far as the flow of information goes, the entire sequence of partial quotients is needed in order to generate a single member in the sequence of approximation coefficients. In 1978, Jurkat and Peyerimhoff [5] showed that for all irrational numbers and for all $n \geq 1$, the point $\left(\theta_{n-1}, \theta_{n}\right)$ lies in the interior of the triangle with vertices $(0,0),(0,1)$ and $(1,0)$. As a result, we have

$$
\theta_{n-1}+\theta_{n}<1
$$

which is an improvement of Vahlen's result (11). In addition, they proved that $a_{n+1}$ can be written as a function of $\left(\theta_{n-1}, \theta_{n}\right)$ but came up short of providing a simple expression that applies to all cases. Combining this observation with the pair of symmetric identities

$$
\theta_{n+1}=\theta_{n-1}+a_{n+1} \sqrt{1-4 \theta_{n-1} \theta_{n}}-a_{n+1}^{2} \theta_{n}, \quad n \geq 1,
$$

and

$$
\theta_{n-1}=\theta_{n+1}+a_{n+1} \sqrt{1-4 \theta_{n+1} \theta_{n}}-a_{n+1}^{2} \theta_{n}, \quad n \geq 1,
$$

due to Dajani and Kraaikamp [4, Proposition 5.3.6], allows us to recover the tail of the sequence of approximation coefficients from a pair of consecutive terms.

We abbreviate the last two equations to the single working formula

$$
\theta_{n \pm 1}=\theta_{n \mp 1}+a_{n+1} \sqrt{1-4 \theta_{n \mp 1} \theta_{n}}-a_{n+1}^{2} \theta_{n}, \quad n \geq 1 .
$$

Our goal, obtained in Theorem [3, is to provide a real valued function $f$ such that $a_{n+1}=f\left(\theta_{n \pm 1}, \theta_{n}\right)$. This will enable us, as expressed in Corollary 4. to eliminate $a_{n+1}$ from formula (44) without disrupting its elegant symmetry. This will enable us to recover the entire sequence $\left\{\theta_{n}\right\}_{0}^{\infty}$ from a pair of consecutive terms. 


\section{Symbolic DYNAMiCS}

The continued fraction expansion is a symbolic representation of irrational numbers in the unit interval as an infinite sequence of positive integers. Let $\lfloor\cdot\rfloor$ be the floor function, whose value on a real number $r$ is the largest integer smaller than or equal to $r$. Then we obtain this expansion for the initial seed $x_{0} \in(0,1)-\mathbb{Q}$ by using the following infinite iteration process:

(1) Let $n:=1$.

(2) Set the reminder of $x_{0}$ at time $n$ to be $r_{n}:=\frac{1}{x_{n-1}} \in(1, \infty)$.

(3) Define the digit and future of $x_{0}$ at time $n$ to be the integer part and fractional part of $r_{n}$ respectively, that is, $a_{n}:=\left\lfloor r_{n}\right\rfloor \in \mathbb{N}$ and $x_{n}:=r_{n}-$ $a_{n} \in(0,1)-\mathbb{Q}$. Increase $n$ by one and go to step $(2)$.

Using this iteration scheme, we obtain

$$
x_{0}=\frac{1}{r_{1}}=\frac{1}{a_{1}+x_{1}}=\frac{1}{a_{1}+\frac{1}{r_{2}}}=\frac{1}{a_{1}+\frac{1}{a_{2}+x_{2}}}=\frac{1}{a_{1}+\frac{1}{a_{2}+\frac{1}{r_{3}}}}=\ldots ;
$$

hence, the quantity $a_{n}$ is no other than the $n^{\text {th }}$ partial quotient of $x_{0}$. We relabel $a_{n}$ as the digit for $x_{0}$ at time $n$ in order to emphasize the underlying dynamical structure at hand and write

$$
x_{0}=\left[r_{1}\right]=\left[a_{1}, r_{2}\right]=\left[a_{1}, a_{2}, r_{3}\right]=\ldots .
$$

The quantity $x_{n}=r_{n}-a_{n}$ is the value of $x_{n-1}$ under the Gauss Map

$$
T:((0,1)-\mathbb{Q}) \rightarrow((0,1)-\mathbb{Q}), \quad T(x):=\frac{1}{x}-\left\lfloor\frac{1}{x}\right\rfloor .
$$

This map is realized as a left shift operator on the set of infinite sequences of digits, i.e.

$$
\left[a_{n}, a_{n+1}, r_{n+2}\right]=x_{n-1} \stackrel{T}{\mapsto} x_{n}=\left[a_{n+1}, r_{n+2}\right], \quad n \geq 1 .
$$

We preserve the $n$ digits that the map $T^{n}$ erases from this symbolic representation of $x_{0}$ by defining the past of $x_{0}$ at time $n \geq 1$ to be

$$
y_{n}:=-a_{n}-\left[a_{n-1}, a_{n-2}, \ldots, a_{1}\right]<-1 .
$$

\section{The natural extension map}

$$
\mathcal{T}(x, y):=\left(\frac{1}{x}-\left\lfloor\frac{1}{x}\right\rfloor, \quad \frac{1}{y}-\left\lfloor\frac{1}{x}\right\rfloor\right)=\left(T(x), \quad \frac{1}{y}-\left\lfloor\frac{1}{x}\right\rfloor\right)
$$

is well defined whenever $x$ is an irrational number and $y<-1$, providing us with the relationship

$$
\left(x_{n+1}, y_{n+1}\right)=\mathcal{T}\left(x_{n}, y_{n}\right), \quad n \geq 1 .
$$

Since $x_{n}$ is uniquely determined by $\left\{a_{n}\right\}_{n+1}^{\infty}$ and $y_{n}$ is uniquely determined by $\left\{a_{n}\right\}_{1}^{n}$, this map can be thought of as one tick of the clock in the symbolic representation of $x_{0}$ using the sequence $\left\{a_{n}\right\}_{1}^{\infty}$ :

$$
\left[\left[a_{1}, a_{2}, \ldots, a_{n} \mid a_{n+1}, a_{n+2}, \ldots\right]\right] \stackrel{\mathcal{T}}{\mapsto}\left[\left[a_{1}, a_{2}, \ldots, a_{n}, a_{n+1} \mid a_{n+2}, \ldots\right]\right],
$$

advancing the present time denoted by $\mid$ one step into the future. 


\section{DYNAMic PAIRS VS. JAGER PAIRS}

Using the dynamical terminology of the last section, we restate Perron's result (2) as

$$
\theta_{n-1}=\frac{1}{x_{n}-y_{n}}, \quad n \geq 1 .
$$

Define the region $\Omega:=(0,1) \times(-\infty,-1) \subset \mathbb{R}^{2}$ and the map

$$
\Psi: \Omega \rightarrow \mathbb{R}^{2}, \quad \Psi(x, y):=\left(\frac{1}{x-y},-\frac{x y}{x-y}\right),
$$

which is clearly well-defined and continuous. Since for all $n \geq 1$, we have $\left(x_{n}, y_{n}\right) \in$ $\Omega$, we use formulas (6) and (8) to obtain

$$
\frac{1}{\theta_{n}}=x_{n+1}-y_{n+1}=\left(\frac{1}{x_{n}}-a_{n+1}\right)-\left(\frac{1}{y_{n}}-a_{n+1}\right)=-\frac{x_{n}-y_{n}}{x_{n} y_{n}}, \quad n \geq 0,
$$

so that

$$
\Psi\left(x_{n}, y_{n}\right)=\left(\theta_{n-1}, \theta_{n}\right) .
$$

We call $\left(\theta_{n-1}, \theta_{n}\right)$ the Jagger Pair of $x_{0}$ at time $n$. We also denote the image $\Psi(\Omega)$ by $\Gamma$. Then

Proposition 1. The set $\Gamma$ is the open region interior to the triangle in $\mathbb{R}^{2}$ with vertices $(0,0),(1,0)$ and $(1,0)$.

Proof. For every positive integer $k \geq 2$, define the open region $\Omega_{k}:=\left(\frac{1}{k}, 1\right) \times$ $(-k,-1)$, whose boundary contains the open line segments $\left(\frac{1}{k}, 1\right) \times\{-1\},\{1\} \times$ $(-k,-1),\left(\frac{1}{k}, 1\right) \times\{-k\}$ and $\left\{\frac{1}{k}\right\} \times(-k,-1)$. Since $\Psi$ is continuous, $\Gamma_{k}:=\Psi\left(\Omega_{k}\right)$ is the open region interior to the image of the boundary for $\Omega_{k}$ under $\Psi$, which we will now find explicitly.

From definition (9) of $\Psi$, we have

$$
x=\frac{1}{u}+y
$$

and

$$
v=-\frac{x y}{x-y}=-u x y .
$$

Set $y:=-1$ and $x \in\left(\frac{1}{k}, 1\right)$ so that, by definition (9) of $\Psi$, we have $u=\frac{1}{x-y}=$ $\frac{1}{x+1} \in\left(\frac{1}{2}, \frac{k}{k+1}\right)$. Formulas (11) and (12) now yield $v=u\left(\frac{1}{u}-1\right)=1-u \in$ $\left(\frac{1}{2}, \frac{1}{k+1}\right)$. Conclude that $\Psi$ maps the open line segment $\left(\frac{1}{k}, 1\right) \times\{-1\}$ in the $x y$ plane to the open line segment between the points $\left(\frac{1}{2}, \frac{1}{2}\right)$ and $\left(\frac{k}{k+1}, \frac{1}{k+1}\right)$ in the $u v$-plane.

Set $x:=1$ and $y \in(-k,-1)$ so that, by definition (9) of $\Psi$, we have $u=\frac{1}{1-y} \in$ $\left(\frac{1}{2}, \frac{1}{k+1}\right)$. Formulas (11) and (12) now yield $v=u\left(\frac{1}{u}-1\right)=1-u \in\left(\frac{1}{2}, \frac{k}{k+1}\right)$. Conclude that $\Psi$ maps the open line segment $\{1\} \times(-k,-1)$ in the $x y$-plane to the open line segment between the points $\left(\frac{1}{2}, \frac{1}{2}\right)$ and $\left(\frac{1}{k+1}, \frac{k}{k+1}\right)$ in the $u v$-plane. 
Set $y:=-k$ and $x \in\left(\frac{1}{k}, 1\right)$, so that, by definition (9) of $\Psi$, we have $u=\frac{1}{x-y}=$ $\frac{1}{x+k} \in\left(\frac{1}{k+1}, \frac{k}{k^{2}+1}\right)$. Formulas (11) and (12) now yield $v=-u\left(\frac{1}{u}-k\right)(-k)=$ $k-k^{2} u \in\left(\frac{k}{k^{2}+1}, \frac{k}{k+1}\right)$. Conclude that $\Psi$ maps the open line segment $(0,1) \times$ $\{-k\}$ in the $x y$-plane to the open line segment between the points $\left(\frac{1}{k+1}, \frac{k}{k+1}\right)$ and $\left(\frac{k}{k^{2}+1}, \frac{k}{k^{2}+1}\right)$ in the $u v$-plane.

Finally, set $x:=\frac{1}{k}$ and $y \in(-k,-1)$, so that, by definition (9) of $\Psi$, we have $u \in\left(\frac{k}{k^{2}+1}, \frac{k}{k+1}\right)$. Formulas (11) and (12) now yield $v=\frac{u}{k}\left(\frac{1}{u}-\frac{1}{k}\right)=\frac{1}{k}-\frac{u}{k^{2}} \in$ $\left(\frac{k}{k^{2}+1}, \frac{k}{k+1}\right)$. Conclude that $\Psi$ maps the open line segment $\left\{\frac{1}{k}\right\} \times(-k,-1)$ in the $x y$-plane to the open line segment between the points $\left(\frac{k}{k^{2}+1}, \frac{k}{k^{2}+1}\right)$ and $\left(\frac{k}{k+1}, \frac{1}{k+1}\right)$ in the $u v$-plane. From the continuity of $\Psi$, we have

$$
\Gamma=\Psi(\Omega)=\Psi\left(\bigcup_{2}^{\infty} \Omega_{k}\right)=\bigcup_{2}^{\infty} \Psi\left(\Omega_{k}\right)=\bigcup_{2}^{\infty} \Gamma_{k} .
$$

Therefore, we conclude the desired result after letting $k \rightarrow \infty$.

Note that since $\left(\theta_{n-1}, \theta_{n}\right)=\Psi\left(x_{n}, y_{n}\right) \in \Gamma$, this observation is in accordance with formula (3).

Lemma 2. The map $\Psi: \Omega \rightarrow \Gamma$ is a homeomorphism with inverse:

$$
\Psi^{-1}(u, v):=\left(\frac{1-\sqrt{1-4 u v}}{2 u},-\frac{1+\sqrt{1-4 u v}}{2 u}\right) .
$$

Proof. First, we will show that $\Psi$ is a bijection. Since the map $\Psi$ is surjective onto its image $\Gamma$, we need only show injectiveness. Let $\left(x_{1}, y_{1}\right),\left(x_{2}, y_{2}\right)$ be two points in $\Omega$ such that

$$
\left(\frac{1}{x_{1}-y_{1}},-\frac{x_{1} y_{1}}{\left(x_{1}-y_{1}\right)}\right)=\Psi\left(x_{1}, y_{1}\right)=\Psi\left(x_{2}, y_{2}\right)=\left(\frac{1}{x_{2}-y_{2}},-\frac{x_{2} y_{2}}{\left(x_{2}-y_{2}\right)}\right) .
$$

By equating the first and then the second components of the exterior terms, we obtain that

$$
x_{1}-y_{1}=x_{2}-y_{2}
$$

and then that $x_{1} y_{1}=x_{2} y_{2}$. Therefore,

$$
\left(x_{1}+y_{1}\right)^{2}=\left(x_{1}-y_{1}\right)^{2}+4 x_{1} y_{1}=\left(x_{2}-y_{2}\right)^{2}+4 x_{2} y_{2}=\left(x_{2}+y_{2}\right)^{2} .
$$

Since both these points are in $\Omega$, they must lie below the line $x+y=0$; hence $x_{1}+y_{1}=x_{2}+y_{2}<0$. Another application of condition (14) now proves that $x_{1}=x_{2}$ and $y_{1}=y_{2}$; hence $\Psi$ is injective.

Since both $\Psi$ and $\Psi^{-1}$ are clearly continuous, it is left to prove that $\Psi^{-1}$ is well-defined and that it is the inverse for $\Psi$. Given $\left(u_{0}, v_{0}\right) \in \Gamma$, set

$$
\left(x_{0}, y_{0}\right):=\Psi^{-1}\left(u_{0}, v_{0}\right)=\left(\frac{1-\sqrt{1-4 u_{0} v_{0}}}{2 u_{0}},-\frac{1+\sqrt{1-4 u_{0} v_{0}}}{2 u_{0}}\right) .
$$

From Proposition 1, we know that $\Gamma$ lies entirely underneath the line $u+v=1$ in the $u v$ plane. The only point of intersection for this line and the hyperbola $4 u v=1$ is the point $(u, v)=\left(\frac{1}{2}, \frac{1}{2}\right)$; hence $\Gamma$ must lie underneath this hyperbola as well. We conclude that $4 u_{0} v_{0}<1$, so that both $x_{0}$ and $y_{0}$ must be real. Another 
implication of the inequality $u+v<1$ is that $4 u v<4 u-4 u^{2}$; hence $1-4 u v>$ $4 u^{2}-4 u+1=(2 u-1)^{2}$. Conclude that $1+\sqrt{1-4 u v}>2 u$, so we must have $y_{0}=-\frac{1+\sqrt{1-4 u_{0} v_{0}}}{2 u_{0}}<-1$.

To prove that $x_{0} \in(0,1)$, we first observe that $\sqrt{1-4 u_{0} v_{0}}<1$ implies that $1-\sqrt{1-4 u_{0} v_{0}}>0$; hence $x_{0}$ is positive. If we further assume by contradiction that $x_{0} \geq 1$, then the definition of $\Psi^{-1}$ (13) will imply the inequality

$$
\begin{aligned}
1+\sqrt{1-4 u_{0} v_{0}} & \leq x_{0}\left(1+\sqrt{1-4 u_{0} v_{0}}\right) \\
& =\frac{1}{2 u_{0}}\left(1-\sqrt{1-4 u_{0} v_{0}}\right)\left(1+\sqrt{1-4 u_{0} v_{0}}\right)=2 v_{0},
\end{aligned}
$$

so that we obtain the inequality

$$
4 v_{0}^{2}-4 v_{0}+1=\left(2 v_{0}-1\right)^{2} \geq 1-4 u_{0} v_{0} .
$$

After the appropriate cancellations and rearrangements, we obtain the inequality $u_{0}+v_{0} \geq 1$, which is in contradiction to Proposition 1. Conclude that $\left(x_{0}, y_{0}\right) \in \Omega$ and $\Psi^{-1}: \Gamma \rightarrow \Omega$ is well-defined.

Finally, we will show that $\Psi^{-1}$ is the inverse for $\Psi$. Let $(u, v) \in \Gamma$ and set $(x, y):=\Psi^{-1}(u, v) \in \Omega$. Using the definitions (9) and (13) of $\Psi$ and $\Psi^{-1}$, the first component of $\Psi(x, y)$ is

$$
\frac{1}{x-y}=\left(\frac{1-\sqrt{1-4 u v}}{2 u}+\frac{1+\sqrt{1-4 u v}}{2 u}\right)^{-1}=\left(\frac{2}{2 u}\right)^{-1}=u,
$$

and its second component is

$$
-\frac{x y}{(x-y)}=-u(x y)=u\left(\frac{1}{4 u^{2}}\left(1-\sqrt{1-4 u v}^{2}\right)\right)=\frac{1}{4 u} \cdot 4 u v=v ;
$$

hence $\Psi^{-1}$ is the right inverse for $\Psi$. Since $\Psi$ is a bijection, we conclude it is the (two-sided) inverse for $\Psi$, completing the proof.

\section{Result}

Theorem 3. Let $x_{0}$ be an irrational number in the unit interval and let $n \in \mathbb{N}$. If $a_{n+1}$ is the digit at time $n+1$ in the continued fraction expansion for $x_{0}$ and if $\left(\theta_{n-1}, \theta_{n}, \theta_{n+1}\right)$ are the approximation coefficients for $x_{0}$ at time $n-1, n$ and $n+1$, then

$$
a_{n+1}=\left\lfloor\frac{1+\sqrt{1-4 \theta_{n-1} \theta_{n}}}{2 \theta_{n}}\right\rfloor=\left\lfloor\frac{1+\sqrt{1-4 \theta_{n+1} \theta_{n}}}{2 \theta_{n}}\right\rfloor .
$$

Proof. Let $\left(x_{n}, y_{n}\right)$ be the dynamic pair of $x_{0}$ at time $n$. Formula (10), the fact that $\Psi$ is a homeomorphism and definition (13) of $\Psi^{-1}$ yield

$$
\left(x_{n}, y_{n}\right)=\Psi^{-1}\left(\theta_{n-1}, \theta_{n}\right)=\left(\frac{1-\sqrt{1-4 \theta_{n-1} \theta_{n}}}{2 \theta_{n-1}},-\frac{1+\sqrt{1-4 \theta_{n-1} \theta_{n}}}{2 \theta_{n-1}}\right) .
$$

Using formula (51), we write $x_{n}=\left[a_{n+1}, r_{n+2}\right]=\frac{1}{a_{n+1}+\left[r_{n+2}\right]}$, so that the first components in the exterior terms of formula (16) equate to

$$
a_{n+1}+\left[r_{n+2}\right]=\frac{2 \theta_{n-1}}{1-\sqrt{1-4 \theta_{n-1} \theta_{n}}}=\frac{1+\sqrt{1-4 \theta_{n-1} \theta_{n}}}{2 \theta_{n}} .
$$


But since $\left[r_{n+2}\right]=x_{n+1}<1$, we have

$$
a_{n+1}=\left\lfloor a_{n+1}+\left[r_{n+2}\right]\right\rfloor=\left\lfloor\frac{1+\sqrt{1-4 \theta_{n-1} \theta_{n}}}{2 \theta_{n}}\right\rfloor,
$$

which is the first equality in (15).

Next, we equate the second components in the exterior terms of formula (16), which, after using formula (7), yields

$$
a_{n}+\left[a_{n-1}, \ldots, a_{1}\right]=\frac{1+\sqrt{1-4 \theta_{n-1} \theta_{n}}}{2 \theta_{n-1}} .
$$

But since $\left[a_{n-1}, \ldots, a_{1}\right]<1$, we conclude that

$$
a_{n}=\left\lfloor a_{n}+\left[a_{n-1}, \ldots, a_{1}\right]\right\rfloor=\left\lfloor\frac{1+\sqrt{1-4 \theta_{n-1} \theta_{n}}}{2 \theta_{n-1}}\right\rfloor .
$$

Adding one to all indices establishes the equality of the exterior terms in (15) and completes the proof.

As a direct consequence of this theorem and formula (44), we obtain:

Corollary 4. Assuming the hypothesis of the theorem, we have

$$
\theta_{n \pm 1}=\theta_{n \mp 1}+\left\lfloor\frac{1+\sqrt{1-4 \theta_{n \mp 1} \theta_{n}}}{2 \theta_{n}}\right\rfloor \sqrt{1-4 \theta_{n \mp 1} \theta_{n}}-\left\lfloor\frac{1+\sqrt{1-4 \theta_{n \mp 1} \theta_{n}}}{2 \theta_{n}}\right\rfloor^{2} \theta_{n} .
$$

\section{ACKNOWLEDGMENTS}

This paper is a development of part of the author's Ph.D. dissertation at the University of Connecticut. Benefiting tremendously from the patience and rigor of his advisor, Andrew Haas, the author would like to thank him for all his efforts. In addition, the author would like to extend his gratitude to Alvaro Lozano-Robledo for his suggestions and corrections.

\section{REFERENCES}

[1] F. Bagemihl and J.R. McLaughlin, Generalization of some classical theorems concerning triples of consecutive convergents to simple continued fractions, J. Reine Angew. Math. 221, 1966. MR0183999 (32 \#1475)

[2] E. B. Burger, Exploring the number jungle: A journey into diophantine analysis, Providence, RI: Amer. Math. Soc., 2000. MR1774066 (2001h:11001)

[3] T. W. Cusick and M. E. Flahive, The Markoff and Lagrange spectra, Math. Surveys and Monograms, no. 30, Amer. Math. Soc., 1989. MR.1010419 (90i:11069)

[4] K. Dajani and C. Kraaikamp, Ergodic theory of numbers, The Carus Math. Monograms, no. 29, Math. Assoc. Amer., 2002. MR1917322 (2003f:37014)

[5] W. B. Jurkat and A. Peyerimhoff, Characteristic approximation properties of quadratic irrationals, Internat. J. Math. \& Math. Sci.(1), 1978. MR0517950 (80a:10050)

[6] O. Perron, Über die approximation irrationaler zahlen durch rationale, Heindelberg Akad. Wiss. Abh.(4), 1921.

[7] J. Tong, The conjugate property of the Borel theorem on diophantine approximation, Math. Z. 184(2), 1983. MR0716268 (85m:11039)

Department of Mathematics. Saint Mary's College of Maryland, Saint Mary's City, MARYLAND 20686

E-mail address: abourla@smcm.edu

Current address: Department of Mathematics and Statistics, American University, 4400 Massachusetts Avenue, NW, Washington, DC 20016

E-mail address: bourla@american.edu 